\title{
Azithromycin for treatment of hospitalised COVID-19 patients: a randomised, multicentre, open-label clinical trial (DAWn-AZITHRO)
}

\author{
Iwein Gyselinck $\mathbb{1}^{1}$, Laurens Liesenborghs ${ }^{2}$, Ann Belmans ${ }^{3}$, Matthias M. Engelen ${ }^{4}$, Albrecht Betrains ${ }^{5,6}$, \\ Quentin Van Thillo (1) ${ }^{7,8}$, Pham Anh Hong Nguyen ${ }^{9}$, Pieter Goeminne ${ }^{10}$, Ann-Catherine Soenen ${ }^{11}$, \\ Nikolaas De Maeyer ${ }^{12}$, Charles Pilette ${ }^{13}$, Emmanuelle Papleux ${ }^{14}$, Eef Vanderhelst $\mathbb{B}^{15}$, \\ Aurélie Derweduwen ${ }^{16}$, Patrick Alexander ${ }^{17}$, Bernard Bouckaert $\mathbb{D}^{18}$, Jean-Benoît Martinot ${ }^{19}$, \\ Lynn Decoster ${ }^{20}$, Kurt Vandeurzen ${ }^{21}$, Rob Schildermans ${ }^{22}$, Peter Verhamme $\mathbb{B}^{23}$, Wim Janssens $\mathbb{( B D}^{1}$ and \\ Robin Vos $\mathbb{1}^{1}$ for the DAWn-AZITHRO investigators ${ }^{24}$
}

\begin{abstract}
${ }^{1}$ Dept of Respiratory Diseases, UZ Leuven and CHROMETA, Research group BREATHE, KU Leuven, Leuven, Belgium. ${ }^{2}$ Laboratory of Virology and Chemotherapy, Dept of Microbiology, Immunology and Transplantation, Rega Institute for Medical Research, KU Leuven, Leuven, Belgium. ${ }^{3}$ Leuven Biostatistics and Statistical Bioinformatics Centre (L-BioStat), KU Leuven, Leuven and University Hasselt, Hasselt, Belgium. ${ }^{4}$ Dept of Cardiovascular Diseases, UZ Leuven and Centre for Molecular and Vascular Biology, KU Leuven, Leuven, Belgium. ${ }^{5}$ Dept of General Internal Medicine, University Hospitals Leuven, Leuven, Belgium. ${ }^{6}$ Dept of Microbiology, Immunology, and Transplantation, KU Leuven, Leuven, Belgium. ${ }^{7}$ Center for Cancer Biology, Vlaams Instituut voor Biotechnologie (VIB), Leuven, Belgium. ${ }^{8}$ Center for Human Genetics, KU Leuven, Leuven, Belgium. ${ }^{9}$ Dept of Respiratory Diseases, Onze Lieve Vrouw Ziekenhuis, Aalst, Belgium. ${ }^{10}$ Dept of Respiratory Diseases, AZ Nikolaas, Sint-Niklaas, Belgium. ${ }^{11}$ Dept of Respiratory Diseases, Jan Yperman Ziekenhuis, Ypres, Belgium. ${ }^{12}$ Dept of Respiratory Diseases, Heilig Hart Ziekenhuis, Leuven, Belgium. ${ }^{13}$ Dept of Respiratory Diseases, Cliniques Universitaires Saint-Luc, Brussels, Belgium. ${ }^{14}$ Dept of Respiratory Diseases, Hôpitaux Iris Sud, Brussels, Belgium. ${ }^{15}$ Dept of Respiratory Diseases, University Hospital UZ Brussel, Vrije Universiteit Brussel, Brussels, Belgium. ${ }^{16}$ Dept of Respiratory Diseases, AZ Klina, Brasschaat, Belgium. ${ }^{17}$ Dept of Respiratory Diseases, AZ Glorieux, Ronse, Belgium. ${ }^{18}$ Dept of Respiratory Diseases, AZ Delta, Roeselare, Belgium. ${ }^{19}$ Dept of Respiratory Diseases, Centre Hospitalier Universitaire-UC Louvain, Namur, Belgium. ${ }^{20}$ Dept of Respiratory Diseases, AZ Turnhout, Turnhout, Belgium. ${ }^{21}$ Dept of Respiratory Diseases, Mariaziekenhuis Noord Limburg, Pelt, Belgium. ${ }^{22}$ Dept of Respiratory Diseases, AZ Sint Lucas, Bruges, Belgium. ${ }^{23}$ Dept of Cardiovascular Diseases, UZ Leuven and Centre for Molecular and Vascular Biology, KU Leuven, Leuven, Belgium. ${ }^{24}$ For a list of the DAWn-AZITHRO investigators, see the Acknowledgements.
\end{abstract}

Corresponding author: Iwein Gyselinck (iwein.gyselinck@kuleuven.be)

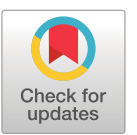

Copyright @The authors 2022

This version is distributed under the terms of the Creative Commons Attribution Non-Commercial Licence 4.0. For commercial reproduction rights and permissions contact permissions@ersnet.org

Received: 28 Oct 2021 Accepted: 28 Dec 2021

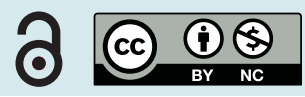

Shareable abstract (@ERSpublications)

Previous randomised controlled studies with azithromycin in hospitalised COVID-19 patients assessed end-points at fixed timepoints. Complementary to this, DAWn-AZITHRO assessed time to sustained improvement. No benefit of azithromycin was shown. https://bit.ly/3FapyC7

Cite this article as: Gyselinck I, Liesenborghs L, Belmans A, et al. Azithromycin for treatment of hospitalised COVID-19 patients: a randomised, multicentre, open-label clinical trial (DAWn-AZITHRO). ERJ Open Res 2022; 8: 00610-2021 [DOI: 10.1183/23120541.00610-2021].

\section{Abstract}

Background and objectives Azithromycin was rapidly adopted as a repurposed drug to treat coronavirus disease 2019 (COVID-19) early in the pandemic. We aimed to evaluate its efficacy in patients hospitalised for COVID-19.

Methods In a series of randomised, open-label, phase 2 proof-of-concept, multicentre clinical trials (Direct Antivirals Working against the novel coronavirus (DAWn)), several treatments were compared with standard of care. In 15 Belgian hospitals, patients hospitalised with moderate to severe COVID-19 were allocated 2:1 to receive standard of care plus azithromycin or standard of care alone. The primary outcome was time to live discharge or sustained clinical improvement, defined as a two-point improvement on the World Health Organization (WHO) ordinal scale sustained for at least 3 days.

Results Patients were included between April 22 and December 17, 2020. When 15-day follow-up data were available for 160 patients (56\% of preset cohort), an interim analysis was performed at request of the independent Data Safety and Monitoring Board. Subsequently, DAWn-AZITHRO was stopped for futility. In total, 121 patients were allocated to the treatment arm and 64 patients to the standard-of-care arm. We 
found no effect of azithromycin on the primary outcome with a hazard ratio of 1.044 (95\% CI 0.772 1.413; $\mathrm{p}=0.7798$ ). None of the predefined subgroups showed significant interaction as covariates in the Fine-Gray regression analysis. No benefit of azithromycin was found on any of the short- and longer-term secondary outcomes.

Conclusion Time to clinical improvement is not influenced by azithromycin in patients hospitalised with moderate to severe COVID-19.

Introduction

Background

The high disease burden and the rapid spread of the severe acute respiratory syndrome coronavirus 2 (SARS-CoV-2) pandemic immediately directed the search for disease-modifying agents towards the repurposing of existing molecules. The Belgian Direct Antivirals Working against the novel Coronavirus studies were a parallel series of proof-of-concept trials, assessing several treatment strategies for hospitalised patients with coronavirus disease 2019 (COVID-19): itraconazole [1], azithromycin [2], anakinra combined with intensified anticoagulation [3] and convalescent plasma [4].

Azithromycin is a macrolide antibiotic of which the use in COVID-19 was encouraged by a documented in vitro antiviral activity against SARS-CoV-2 and other viruses [5, 6], previous clinical experiences with influenza [7, 8] and data from uncontrolled studies early during the pandemic [9, 10]. Apart from its antibiotic and potential antiviral properties, it was hypothesised that azithromycin's broad range of immunomodulatory effects could temper COVID-19-induced hyperinflammation [11].

Until now, primary end-points that have been assessed in randomised controlled trials with azithromycin in hospitalised COVID-19 patients are clinical status at day 15 [12, 13] and 28-day mortality [14, 15]. No benefit of azithromycin was shown for any of these outcomes.

Objectives

In this randomised controlled trial, we assessed the effect of azithromycin on time to discharge or sustained clinical improvement, based on the World Health Organization (WHO) issued ordinal scale.

Materials and methods

Trial design

DAWn-AZITHRO was an open-label, randomised, adaptive clinical trial conducted in 15 Belgian hospitals and coordinated by University Hospitals Leuven to assess if azithromycin added to standard of care could shorten time to discharge or clinical improvement in hospitalised COVID-19 patients. The study protocol and statistical analysis plan are available in the supplementary appendix, and have been published previously [2]. The study was approved by a central Ethics Committee (Comité d'Éthique HospitaloFacultaire de Liège) and the Federal Agency for Medicines and Health Products, and registered in the EU Clinical Trial Register (EudraCT: 2020-001614-38A). The study was conducted and monitored in accordance with the Good Clinical Practice guidelines of the International Conference on Harmonisation of technical requirements for registration of pharmaceuticals for human use (ICH-GCP) guidelines. An independent Data Safety and Monitoring Board (DSMB) assessed the trial progress and patient safety and wellbeing. The DSMB performed pre-planned safety reviews for 80 and 160 patients, and could perform ad hoc analyses in case of substantial evidence of a safety issue.

\section{Participants}

Adults ( $\geqslant 18$ years) hospitalised on a dedicated COVID-19 ward were eligible if they had symptomatic illness of any duration with (1a) radiographic infiltrates or (1b) clinical signs of pneumonia with an oxygen saturation of $\leqslant 94 \%$ on room air or required respiratory support, and (2a) recent laboratory or (2b) radiographic confirmed diagnosis of COVID-19 ( $\leqslant 72 \mathrm{~h}$ before randomisation).

Exclusion criteria were elevated liver transaminases (AST or ALT >5 times the upper limit of normal), pregnancy or breastfeeding, allergy to macrolides, any medical condition which would impose an unacceptable safety hazard by participation to the study, heart failure with severely reduced ejection fraction ( $\leqslant 30 \%$ ), a prolonged corrected QT interval on electrocardiogram ( $>470 \mathrm{~ms}$ for males and $>480 \mathrm{~ms}$ for females) and the use of macrolides during the last week prior to admission.

Informed consent was obtained prior to randomisation. When written informed consent was not obtainable due to restrictions for research staff to access the isolation ward, oral consent was documented in the electronic medical record, and completed with a signed consent as soon as possible. If patients were unable to provide consent, the legal representative was consulted instead. 


\section{Randomisation}

Eligible and consenting patients were randomly allocated to azithromycin on top of standard of care (intervention group) or standard of care alone (control group) according to a 2:1 allocation scheme stratified by study site, using randomly selected block sizes of 6 or 9 . Randomisation was done using a centralised web-based randomisation application.

\section{Blinding}

The study was open label. Patients, clinicians and study personnel were aware of the assigned treatment. The trial statistician was not given access to the full database and was not aware of the allocated treatments. The trial statistician remained blinded until database lock.

\section{Interventions}

Intervention

In the intervention group, $500 \mathrm{mg}$ of azithromycin was given on top of standard of care for the first 5 consecutive days, administered once daily as oral tablets or syrup. Standard-of-care treatment followed regularly updated national and international recommendations, as allowed by the adaptive design. ECG was monitored in patients at risk for long QT. When QTc exceeded $500 \mathrm{~ms}$ and/or QTc increased by $>60$ ms compared to baseline, azithromycin was interrupted or discontinued.

\section{Data collection}

We collected patients' demographics and clinical data, including medical history, use of concomitant therapies, clinical investigation and National Early Warning Score (NEWS), laboratory results and radiographic investigations. Clinical status was assessed daily until discharge and on day 15, day 29 and the follow-up visit 5-7 weeks after discharge.

\section{Outcomes}

The primary outcome was the time from randomisation to alive discharge or sustained clinical improvement, the latter defined as an improvement of $>2$ points compared to the highest value of day 0 and 1 and sustained for at least 3 days. The clinical status was recorded on a 7-point ordinal scale: 1) not hospitalised, no limitations on activities; 2) not hospitalised, limitation on activities; 3) hospitalised, not requiring supplemental oxygen; 4) hospitalised, requiring supplemental oxygen; 5) hospitalised, on noninvasive ventilation or high-flow oxygen devices; 6) hospitalised, on invasive mechanical ventilation or extracorporeal membrane oxygenation; and 7) death.

Secondary outcomes were daily clinical status on the ordinal scale while hospitalised and on days 15 and 29, daily NEWS while hospitalised and on days 15 and 29, cumulative clinical status up to day 15 (i.e. sum of daily clinical status scores from days 1 to 15), mortality on day 15 and day 29, time to intensive care unit (ICU) admission, time to death, duration of supplemental oxygen, duration of mechanical ventilation, duration of hospitalisation, duration of intensive care stay, adverse events graded as 4 or 5 or severe adverse events (SAEs), QTc abnormalities and a combined cardiac end-point (hs-troponin T levels $>0.5 \mathrm{ng} \cdot \mathrm{mL}^{-1}$ and/or ventricular arrhythmia requiring intervention and/or sudden cardiac death).

\section{Sample size}

Based on the trial of CAO et al. [16], we assumed that $40 \%$ of patients would have reached a 2-point improvement on the ordinal scale with standard of care at day 15. Using a log-rank test, with a two-sided significance level of $5 \%$ and a power of $80 \%$ and using a (2:1) randomisation ratio in favour of azithromycin, we estimated that a total sample size of 269 patients was required to detect an absolute improvement of $17.5 \%$. Taking early dropouts into account, we decided on a total sample size of 282 patients.

\section{Statistical methods}

A detailed description of the analysis is provided in the statistical analysis plan, which was finalised and filed before database lock (supplementary appendix). A brief summary is provided here.

Analysis sets were finalised during a Blind Review Meeting prior to database lock. The full analysis set (FAS) included all randomised patients, except patients that were confirmed to be SARS-CoV-2 negative, and patients who withdrew consent to use any data immediately after randomisation and before treatment administration. The per protocol set (PPS) included all FAS patients, but patients randomised to azithromycin who missed 2 or more days of dosing were excluded. The primary analysis set of interest was the FAS. All efficacy analyses were repeated on the PPS as a sensitivity analysis. 
Missing clinical status data were accounted for by means of multiple imputation, using a total of 100 imputations [17]. Treatment effects for all end-points were estimated by an appropriate measure and presented with 95\% confidence intervals and were adjusted for study site (small versus large sites, according to median size) and period (1st and 2nd wave). The primary end-point was compared using competing risk methodology, using cumulative incidence functions to estimate event rates and a Fine \& Gray regression model to obtain cause-specific hazard ratios. Daily clinical status was analysed as a proportional odds logistic regression to estimate the common odds ratio. All-cause mortality and survival without mechanical ventilation up to 30 days were assessed using a Cox regression to obtain hazard ratios. Incidence rates were estimated using Kaplan-Meier methodology. Time to hospital discharge, incidence and duration of supplemental oxygen, mechanical ventilation and ICU were analysed using the same methodology as for the primary end-point. Cumulative clinical status scores were analysed using a general linear model on the log-transformed scores to obtain a treatment ratio of geometric means between the treatment groups.

Prespecified subgroup analyses were performed for the primary end-point, considering the following subgroups: duration of symptoms prior to enrolment (according to observed median); age groups (according to observed median); study period; clinical status at baseline (3 and 4 versus 5 and 6) and ethnicity.

All tests were two-sided and assessed at a significance level of 5\%. No correction was made for multiple secondary end-points. All analyses were performed using SAS software version 9.4 for Windows 10 (SAS Institute, Cary, NC, USA).

\section{Interim analysis and early trial termination}

No safety issues were raised during the pre-planned safety reviews. Online preprint publication of data regarding azithromycin from the RECOVERY-trial [14], which failed to demonstrate a benefit of azithromycin, became available just prior to completion of our 15-day follow-up of the first 160 included patients. Therefore, the trial steering committee and DSMB recommended to additionally perform a futility analysis on these patients, included in the second safety review. Recruitment was halted on December 18, 2020, awaiting the results; 185 patients were randomised at that time. Eventually, the trial was stopped for futility on January 25, 2021, as the conditional power to detect a significant difference for the primary outcome was $0.4 \%$ (futility analysis and DSMB recommendations are included in the supplementary appendix).

Results

\section{Patients}

Between April 24, 2020 and December 17, 2021, 185 patients were allocated to azithromycin (n=121) or standard of care $(n=64)$. Two patients in the intervention group were excluded from the FAS ( $n=119$, equals safety set (SS)), as they withdrew consent and were not treated according to allocation (consort diagram: supplementary figure S1). Baseline data are presented in table 1 and were well matched between intervention and standard of care (SOC) group. Participants had a mean \pm sD age of $62 \pm 15$ years; $37.8 \%$ of them were women. $16.9 \%$ had a history of diabetes and $44.8 \%$ a history of arterial hypertension. $40.0 \%$ were current or former smokers.

During hospitalisation, $14.9 \%$ of patients received hydroxychloroquine. There was a nonsignificant trend towards increased use in the SOC group. Use of other concomitant medication was similar between both groups, with over half of patients receiving systemic corticosteroids (supplementary table S1).

140 patients (76.5\%) presented at the ambulatory pulmonology clinic for a follow-up visit 5-7 weeks after discharge. Data on exploratory outcomes were collected at that timepoint, such as diffusion capacity for carbon monoxide ( $\mathrm{n}=137)$, high-resolution computed tomography (CT) scan $(n=115)$ and 6-min walk distance $(n=121)$.

\section{Primary outcome}

In the azithromycin group and the SOC group, respectively, the primary outcome was met in 39.5\% versus $35.9 \%$ at day $5,78.2 \%$ versus $81.3 \%$ at day 15 and $86.6 \%$ versus $89.1 \%$ at day 29 . Median time to live discharge or sustained clinical improvement in azithromycin versus SOC group was 6 days versus 8 days. No significant difference between treatments was found (subdistribution hazard ratio (HR) 1.023; 95\% CI 0.758-1.379; $\mathrm{p}=0.8839$; figure 1). No statistically significant interactions or treatment effects could be observed for any of the predefined subgroups (figure 2). 
TABLE 1 Subject disposition and baseline information: full analysis set/safety set

\begin{tabular}{|c|c|c|c|c|}
\hline Full analysis set=safety set & Statistic & AZITHRO + SOC & SOC & Total \\
\hline Total number of patients & $\mathrm{n}$ & 119 & 64 & 183 \\
\hline \multicolumn{5}{|l|}{ Demographics } \\
\hline Age years & Mean \pm SD & $63 \pm 15$ & $59 \pm 15$ & $62 \pm 15$ \\
\hline Female & $\mathrm{n} / \mathrm{N}(\%)$ & $40 / 119(33.61)$ & $30 / 64(46.88)$ & $70 / 183(38.25)$ \\
\hline \multicolumn{5}{|l|}{ Ethnicity } \\
\hline Caucasian & $\mathrm{n} / \mathrm{N}(\%)$ & $104 / 119(87.39)$ & $55 / 64(85.94)$ & $159 / 183(86.89)$ \\
\hline North Africa and Middle East & $\mathrm{n} / \mathrm{N}(\%)$ & $11 / 119(9.24)$ & $5 / 64(7.69)$ & $16 / 183(8.74)$ \\
\hline Black or sub-Sahara (Africa) & $\mathrm{n} / \mathrm{N}(\%)$ & $3 / 119(2.52)$ & $1 / 64(1.56)$ & $4 / 183(2.19)$ \\
\hline Other & $\mathrm{n} / \mathrm{N}(\%)$ & $1 / 119(0.84)$ & $3 / 64(4.68)$ & $4 / 183(2.19)$ \\
\hline \multicolumn{5}{|l|}{ Medical history } \\
\hline Diabetes mellitus & $\mathrm{n} / \mathrm{N}(\%)$ & $22 / 119(18.49)$ & 9/64 (14.06) & $31 / 183(16.94)$ \\
\hline Arterial hypertension & $\mathrm{n} / \mathrm{N}(\%)$ & $55 / 119(46.22)$ & $27 / 64(42.19)$ & $82 / 183(44.81)$ \\
\hline Arrhythmia & $\mathrm{n} / \mathrm{N}(\%)$ & $19 / 119(15.97)$ & $12 / 64(18.75)$ & $31 / 183(16.94)$ \\
\hline \multicolumn{5}{|l|}{ Smoking status } \\
\hline Active & $\mathrm{n} / \mathrm{N}(\%)$ & $5 / 110(4.55)$ & $4 / 55(7.27)$ & 9/165 (5.45) \\
\hline Former & $\mathrm{n} / \mathrm{N}(\%)$ & $37 / 110(33.64)$ & $20 / 55(36.36)$ & $57 / 165(34.55)$ \\
\hline Never & $\mathrm{n} / \mathrm{N}(\%)$ & $68 / 110(61.82)$ & $31 / 55(56.36)$ & $99 / 165(60.00)$ \\
\hline Chronic pulmonary disease ${ }^{\#}$ & $\mathrm{n} / \mathrm{N}(\%)$ & $4 / 119(3.36)$ & $2 / 63(3.17)$ & $6 / 182(3.30)$ \\
\hline COPD & $\mathrm{n} / \mathrm{N}(\%)$ & $7 / 119(5.88)$ & $8 / 64(12.50)$ & $15 / 183(8.20)$ \\
\hline Asthma & $\mathrm{n} / \mathrm{N}(\%)$ & $10 / 119(8.40)$ & $5 / 64(7.81)$ & $15 / 183(8.20)$ \\
\hline Heart failure & $\mathrm{n} / \mathrm{N}(\%)$ & $8 / 119(6.72)$ & $3 / 64(4.69)$ & $11 / 183(6.01)$ \\
\hline Ischaemic heart disease & $\mathrm{n} / \mathrm{N}(\%)$ & $10 / 119(8.40)$ & $8 / 64(12.50)$ & $18 / 183(9.84)$ \\
\hline Chronic kidney disease & $\mathrm{n} / \mathrm{N}(\%)$ & $10 / 119(8.40)$ & $6 / 64(9.38)$ & $16 / 183(8.74)$ \\
\hline \multicolumn{5}{|l|}{ Respiratory status at first presentation } \\
\hline Signs of respiratory distress at first & $\mathrm{n} / \mathrm{N}(\%)$ & $57 / 119(47.90)$ & $33 / 61(54.10)$ & $90 / 180(50.00)$ \\
\hline
\end{tabular}

presentation (i.e. oxygen saturation $<93 \%, P_{\mathrm{aO}_{2}} / F_{\mathrm{IO}_{2}}<300 \mathrm{mmHg}$, respiratory rate $>30$ breaths $\cdot \mathrm{min}^{-1}$ )

Respiratory support within first $2 \mathrm{~h}$

Oxygen support (oxygen mask or nasal prongs)

High-flow oxygen support or noninvasive ventilation

Mechanical ventilation ECMO

$\mathrm{n} / \mathrm{N}(\%)$

$\mathrm{n} / \mathrm{N}(\%)$

89/119 (74.79)

$46 / 63$ (73.02)

$135 / 182(74.18)$

Clinical status at baseline

3) Hosp., not requiring supplemental oxygen

4) Hosp., requiring supplemental oxygen

5) Hosp., on noninvasive ventilation

6) Hosp., on invasive MV or ECMO

7) Death

$\mathrm{n} / \mathrm{N}(\%)$

$\mathrm{n} / \mathrm{N}(\%)$

$5 / 119(4.20)$

$4 / 64(6.25)$

9/183 (4.92)

$\mathrm{n} / \mathrm{N}(\%)$

$0 / 119(0.00)$

$3 / 64$ (4.69)

$0 / 64(0.00)$

$3 / 183(1.64)$

$1 / 119(0.84)$

$1 / 183(0.55)$

Laboratory parameters at baseline

CRP $\mathrm{mg} \cdot \mathrm{L}^{-1}$

$\mathrm{n} / \mathrm{N}(\%)$

22/119 (18.49)

$14 / 64(21.88)$

36/183 (19.67)

$\mathrm{n} / \mathrm{N}(\%)$

88/119 (73.95)

43/64 (67.19)

$131 / 183(71.58)$

WBC $10^{9} / \mathrm{L}$

Ref. $\leqslant 5$

Ref. 4.0-10.0

$\mathrm{n} / \mathrm{N}(\%)$

$\mathrm{n} / \mathrm{N}(\%)$

9/119 (7.56)

$0 / 119(0.00)$

$\mathrm{n} / \mathrm{N}(\%)$

$0 / 119(0.00)$

$5 / 64(7.81)$

2/64 (3.13)

$14 / 183(7.65)$

$0 / 64(0.00)$

$2 / 183(1.09)$

$0 / 183(0.00)$

Lymphocytes $10^{9} / \mathrm{L}$

Neutrophils $10^{9} / \mathrm{L}$

Ferritin $\mu \mathrm{g} \cdot \mathrm{L}^{-1}$

D-dimer $\mu \mathrm{g} \cdot \mathrm{L}^{-1}$

Median (Q1-Q3)

$73.8(35.2-125.8)$

$59.5(23.5-93.3)$

$68.0(33.1-119.2)$

Median (Q1-Q3)

$6.0(4.2-8.0)$

$5.6(4.1-8.0)$

$5.8(4.2-8.0)$

Ref. 2.5-7.8

$1.0(0.7-1.4)$

$1.0(0.7-1.3)$

$1.0(0.7-1.4)$

Median (Q1-Q3)

$4.4(2.9-6.5)$

$4.5(3.0-6.6)$

Fibrinogen $\mathrm{g} \cdot \mathrm{L}^{-1}$

Ref. $30-400$

Median (Q1-Q3)

$4.5(3.4-6.7)$

$736.0(492.0-1259.0)$

Ref. $\leqslant 500 \quad$ Median (Q1-Q3)

$722.5(408.0-1057.0)$

$748.0(529.0-1420.0)$

$723.5(455.5-1160.0)$

Median (Q1-Q3)

$9.3(5.3-547.0)$

Ref. $\geqslant 60 \quad$ Median (Q1-Q3)

$82.0(64.0-90.0)$

$139.2(4.5-547.0)$

$85.0(65.0-90.0)$

ECG at baseline

QTc (MS) (Fridericia formula)

Symptom onset

Time from symptom onset to

n, mean $\pm s D$

$n=111,418.9 \pm 25.3$

$n=57,416.5 \pm 28.6$

$n=168,418.1 \pm 26.4$

randomisation, days

Median (Q1-Q3)

$7(4-10)$

$7(5-10)$

$7(5-10)$

AZITHRO: azithromycin; SOC: standard of care; $P_{\mathrm{aO}}$ : arterial oxygen tension; $F_{\mathrm{IO}_{2}}$ : inspiratory oxygen fraction; ECMO: extracorporeal membrane oxygenation; Hosp.: hospitalised; MV: mechanical ventilation; Ref: reference value; CRP: C-reactive protein; WBC: white blood cell count; eGFR: estimated glomerular filtration rate. \#: not COPD or asthma. 


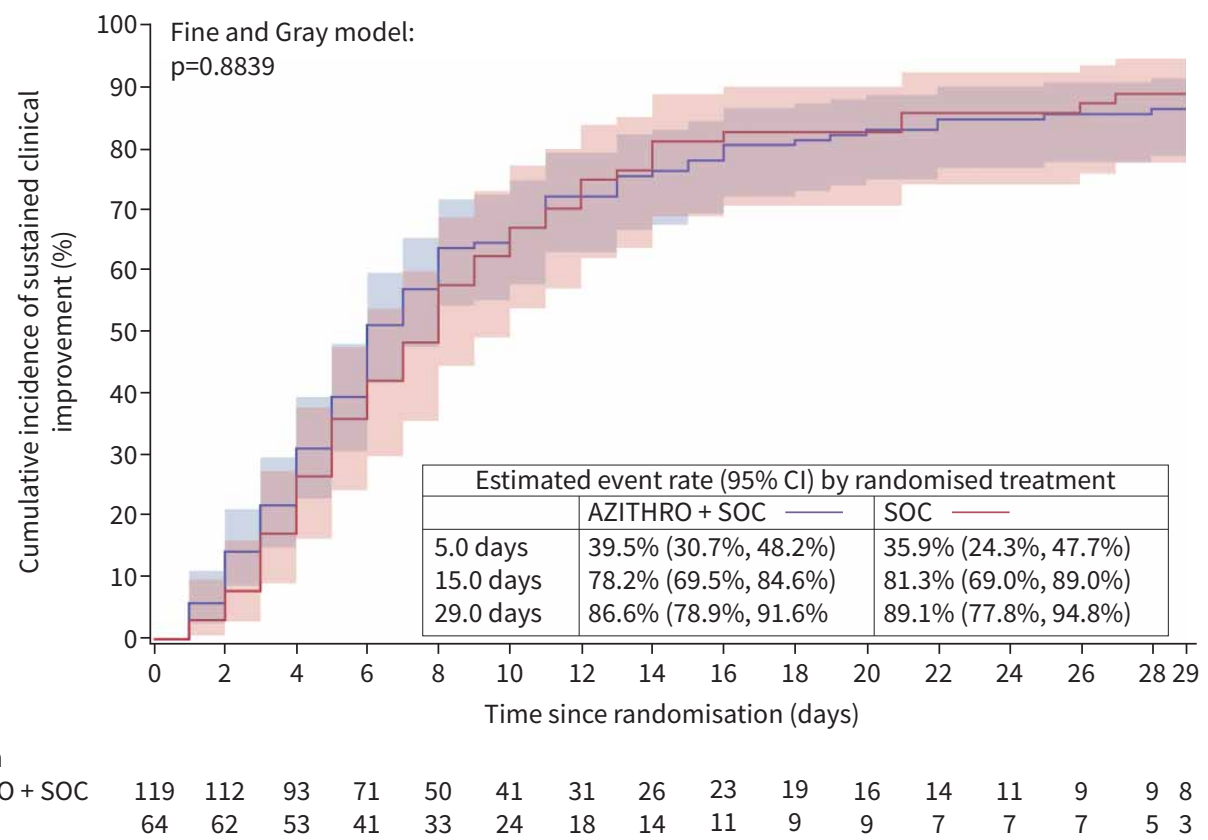

FIGURE 1 Primary end-point: cumulative incidence function of sustained clinical improvement or live discharge (full analysis set). AZITHRO: azithromycin; SOC: standard of care.

\section{Secondary outcomes}

Secondary end-points are shown in table 2 and supplementary tables S2 and S3. Daily clinical status is graphically presented in figure 3. There was no significant difference in the cumulative clinical status at day 15 (geometric mean in azithromycin versus SOC group 42.61 versus 42.60; treatment ratio 1.00; 95\% CI $0.90-1.12$; $p=0.9508$ ) or the odds of having a lower clinical status at day 15 (proportional odds 0.83 ; 95\% CI 0.47-1.53; p=0.5776). Neither was there a difference in all-cause mortality up to day 29 (HR 1.109; 95\% CI 0.339-3.628; $\mathrm{p}=0.8666$ ) or any of the other secondary end-points.

\section{Safety outcomes}

There was a comparable number of QTc abnormalities reported in the azithromycin group versus the SOC group (4 (3.36\%) versus 1 (1.56\%); OR 2.54; 95\% CI 0.27-24.21; p=0.4164; supplementary table S2). Incidence of the combined cardiac safety end-point was similar in both groups $(n=24$ in the azithromycin (20.17\%) versus $\mathrm{n}=12$ in SOC group (18.75\%); OR 1.40; 95\% CI 0.61-3.19; $\mathrm{p}=0.4265$; table 2). Adverse events are summarised in supplementary tables S4-S9.

\section{Long-term outcomes}

In $63 \%$ of patients in both the azithromycin and SOC group, chest CT was performed after 5-7 weeks. There was a similar proportion of patients with normal CTs (25 out of 75 in the azithromycin (33.3\%) versus 12 out of 40 in SOC group (30.0\%); OR 1.56; 95\% CI 0.64-3.79; $\mathrm{p}=0.3233$ ). There was also no significant difference in diffusion capacity (mean transfer factor for carbon monoxide in azithromycin versus SOC group, $66.5 \%$ predicted versus $67 \%$ predicted; treatment difference obtained using general linear model, 0.22\%; 95\% CI -11.1-11.51; p=0.9695), nor the 6-min walk distance (median walking distance in azithromycin versus SOC group $496.5 \mathrm{~m}$ versus $456.3 \mathrm{~m}$; treatment difference obtained using general linear model $32.51 \mathrm{~m}$; 95\% CI $-21.2-86.25$; $\mathrm{p}=0.2383$ ) at 5-7 weeks.

\section{Discussion}

This randomised clinical trial on the use of azithromycin for hospitalised COVID-19 patients was prematurely stopped after 185 patients, which constitutes about $70 \%$ of the prespecified sample size. The results showed no effect of adding azithromycin to the standard of care on clinical outcomes of hospitalised COVID-19 patients. Median time to sustained clinical improvement or live discharge from the hospital was not significantly different in patients that received azithromycin on top of standard of care. Neither was there an observed effect in any of the secondary outcomes. These findings remained consistent across all prespecified subgroups. 


\begin{tabular}{|c|c|c|c|c|c|c|}
\hline Subgroup & $\begin{array}{c}\text { AZITHRO + SOC } \\
\text { Cumulative incidence (\%) } \\
\text { [n] Est. }(95 \% \mathrm{CI})\end{array}$ & $\begin{array}{c}\mathrm{SOC} \\
\text { Cumulative incidence (\%) } \\
{[\mathrm{n}] \text { Est. }(95 \% \mathrm{Cl})}\end{array}$ & $\begin{array}{c}\text { Hazard ratio } \\
\text { versus SOC } \\
(95 \% \mathrm{CI})\end{array}$ & p-value & $\begin{array}{l}\text { SOC } \\
\text { Better }\end{array}$ & $\begin{array}{l}\text { AZITHRO + SOC } \\
\text { Better }\end{array}$ \\
\hline Total population & [119] 86.57 (78.89-91.60) & [64] $89.06(77.83-94.79)$ & $1.02(0.75-1.38)$ & 0.8839 & & 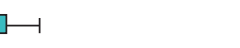 \\
\hline \multicolumn{7}{|c|}{ Time from symptoms to randomisation (interaction: $p=0.6839$ ) } \\
\hline$<7$ days & {$[48] 85.46(70.88-93.08)$} & {$[18] 83.33(52.09-95.03)$} & $1.25(0.73-2.14)$ & 0.4186 & $\mapsto$ & $-\square$ \\
\hline$\geq 7$ days & [69] $86.96(75.95-93.14)$ & [45] $93.33(78.53-98.05)$ & $0.91(0.64-1.31)$ & 0.6202 & $\mapsto$ & $\longrightarrow$ \\
\hline \multicolumn{7}{|l|}{ Age (interaction: $p=0.2067$ ) } \\
\hline$<63$ years & [51] $98.04(78.15-99.84)$ & [35] $91.43(73.80-97.39)$ & $1.46(0.94-2.28)$ & 0.0902 & & $\square$ \\
\hline$\geq 63$ years & {$[68] 77.97(65.90-86.20)$} & [29] $86.21(65.05-95.01)$ & $0.90(0.59-1.35)$ & 0.6004 & $\mapsto \square$ & -1 \\
\hline \multicolumn{7}{|c|}{ Clinical status at baseline (interaction: $p=0.8756$ ) } \\
\hline 3 and 4 & [110] 87.29 (79.31-92.34) & [57] $91.23(79.44-96.40)$ & $0.94(0.68-1.31)$ & 0.7308 & $\mapsto$ & $\longrightarrow$ \\
\hline 5 and 6 & [9] $77.78(28.18-95.14)$ & [7] $71.43(14.98-94.21)$ & $1.35(0.59-3.08)$ & 0.4777 & $\mapsto$ & $\square$ \\
\hline \multicolumn{7}{|l|}{ Study period (interaction: $\mathrm{p}=0.2150$ ) } \\
\hline April 24 to August 8, 2020 & [30] $90.00(68.94-97.06)$ & [21] $100.0(\mathrm{NC}-\mathrm{NC})$ & $0.73(0.46-1.17)$ & 0.1959 & $\mapsto \square$ & -1 \\
\hline August 9 to December 16, 2020 & {$[89] 85.42(76.00-91.34)$} & [43] $83.72(67.98-92.15)$ & $1.19(0.82-1.73)$ & 0.3702 & $\vdash$ & \\
\hline \multicolumn{7}{|c|}{ Prior corticosteroid therapy (interaction: $p=0.3113$ ) } \\
\hline No & [109] $87.16(79.13-92.24)$ & [58] $93.10(81.59-97.52)$ & $0.93(0.69-1.26)$ & 0.6444 & $\mapsto$ & $\mapsto$ \\
\hline Yes & [10] $80.27(31.01-95.96)$ & [6] $50.00(7.01-83.46)$ & $2.54(0.65-9.92)$ & 0.1804 & $\longmapsto$ & 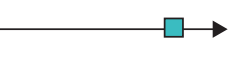 \\
\hline \multicolumn{7}{|l|}{ Ethnicity (interaction: $\mathrm{p}=0.6758$ ) } \\
\hline Caucasian & [104] 86.56 (78.19-91.88) & [55] $87.27(74.47-93.91)$ & $1.00(0.72-1.40)$ & 0.9878 & $\mapsto$ & $\longmapsto$ \\
\hline Non-Caucasian & [15] $86.67(49.27-97.15)$ & [9] 100.0 (NC-NC) & $1.19(0.60-2.36)$ & 0.6145 & $\longmapsto$ & $-\square$ \\
\hline
\end{tabular}

FIGURE 2 Forest plot subgroup analyses for primary end-point: time to sustained clinical improvement or discharge (full analysis set). AZITHRO: azithromycin; SOC: standard of care; NC: not calculated.

A convincing lack of benefit of azithromycin on 28-day mortality in hospitalised patients with COVID-19 was already shown in the RECOVERY-trial [14]. Moreover, CAVALCANTI et al. [12] and FurTADo et al. [13] demonstrated no effect on clinical status at day 15. However, assessment of outcomes at a fixed timepoint, may risk missing the time of clinical benefit [18], and the often-used proportional odds model is difficult to clinically interpret. The primary outcome of DAWn-AZITHRO, a time-to-event-analysis of the WHO-issued ordinal scale, was therefore still relevant and readily interpretable.

A strength of our study was the follow-up after hospital discharge. While the lack of effect of azithromycin on short-term outcomes like 15- and 28-day clinical status and mortality have been extensively published, few have reported on longer-term and functional outcomes. It has been hypothesised that azithromycin treatment in COVID-19 may promote tissue repair and reduce post-COVID fibrosis by modulation of macrophage and myofibroblast function [19]. At our 5- to 7-week follow-up visit, the proportion of patients with complete resolution of COVID-related radiological abnormalities were similar in treated and untreated patients. Correspondingly, we also found no meaningful difference in diffusion capacity nor walking distance. We thus could not demonstrate that azithromycin promotes post-COVID recovery.

There may be several reasons why azithromycin fails to benefit COVID-19 patients. First, the in vitro antiviral activity is weak, compared with direct-acting antivirals like remdesivir [1], and azithromycin has not been shown to aid viral clearance in vivo [20]. Second, the incidence of bacterial superinfection in COVID-19 is low [21], and, contrary to what is seen in influenza [7, 8, 22], a prophylactic effect against post-viral pneumococcal and atypical pneumonia is thus absent. Last, the broad immunomodulatory actions of azithromycin are well known and broadly exploited in day-to-day respiratory care, albeit mostly for long-term treatment of chronic respiratory conditions such as COPD, cystic fibrosis (CF) and non-CF 
TABLE 2 Trial primary and secondary end-points: full analysis set (FAS)

\begin{tabular}{|c|c|c|c|c|c|c|}
\hline \multirow[t]{2}{*}{ Full analysis set $(n=183)$} & \multirow[t]{2}{*}{ Statistic } & \multicolumn{2}{|c|}{ Estimate $(95 \% \mathrm{Cl})$} & \multirow[t]{2}{*}{ Treatment effect } & \multirow[t]{2}{*}{ Estimate $(95 \% \mathrm{Cl})$} & \multirow[t]{2}{*}{$\mathrm{p}$-value } \\
\hline & & AZITHRO & SOC & & & \\
\hline \multicolumn{7}{|l|}{ Primary outcome } \\
\hline $\begin{array}{l}\text { Incidence of clinical improvement or live } \\
\text { discharge }\end{array}$ & & & & Subdistribution $\mathrm{HR}^{\#}$ & $1.023(0.758-1.379)$ & 0.8839 \\
\hline At 15 days & $\mathrm{CIF}^{\bullet}(\%)$ & $78.2(69.5-84.6)$ & $81.3(69.0-89.0)$ & & & \\
\hline At 29 days & $\mathrm{CIF}^{\bullet}(\%)$ & $86.6(78.9-91.6)$ & $89.1(77.8-94.8)$ & & & \\
\hline $\begin{array}{l}\text { Time to sustained clinical improvement or live } \\
\text { discharge }\end{array}$ & Median (days) & $6(6-8)$ & $8(6-10)$ & & & \\
\hline \multicolumn{7}{|c|}{ Secondary outcomes (compared to FAS, total $n$-values may be lower than $n=119$ for AZITHRO and $n=64$ for SOC due to missing values ${ }^{+}$) } \\
\hline Clinical status at day 15 & $\mathrm{n} / \mathrm{N}(\%)$ & & & $\begin{array}{c}\text { Common OR of having lower clinical } \\
\text { status at day } 15^{\S}\end{array}$ & $0.83(0.47-1.53)$ & 0.5776 \\
\hline 1) Not hosp., no limitations & & 14/97 (14.58) & $11 / 53(20.75)$ & & & \\
\hline 2) Not hosp., limitations & & $50 / 97(51.55)$ & 28/53 (52.83) & & & \\
\hline 3) Hosp., no supplemental oxygen & & $7 / 97(7.22)$ & $2 / 53(3.77)$ & & & \\
\hline 4) Hosp., requiring oxygen & & 10/97 (10.31) & $4 / 53(7.55)$ & & & \\
\hline 5) Hosp., noninvasive ventilation & & $5 / 97(5.15)$ & $3 / 53(5.66)$ & & & \\
\hline 6) Hospitalised, MV or ECMO & & $7 / 97(7.22)$ & $4 / 53(7.55)$ & & & \\
\hline 7) Death & & $4 / 97(4.12)$ & $1 / 53(1.89)$ & & & \\
\hline Cumulative clinical status up to day 15 & Geometric mean ${ }^{f}$ & $42.61(39.66-45.77)$ & $42.60(38.64-46.98)$ & Treatment ratio \#\# & $1.00(0.90-1.12)$ & 0.9508 \\
\hline \multicolumn{7}{|l|}{ All-cause mortality } \\
\hline 15 days & KM (\%) & $3.4(1.3-8.7)$ & $1.6(0.2-10.6)$ & $H R^{4 थ}$ & $1.799(0.201-16.09)$ & 0.5996 \\
\hline 29 days & KM (\%) & $7.6(4.0-14.0)$ & $6.5(2.5-16.3)$ & $H R^{थ ฯ}$ & $1.109(0.339-3.628)$ & 0.8666 \\
\hline \multicolumn{7}{|l|}{ ICU (29 days) } \\
\hline Incidence (all patients) ${ }^{\text {बฯฯ }}$ & $\mathrm{CIF}^{थ /}(\%)$ & $26.1(18.5-34.2)$ & $23.4(13.9-34.4)$ & Subdistribution $\mathrm{HR}^{\#}$ & $1.066(0.572-1.985)$ & 0.8412 \\
\hline Duration of ICU stay (ICU admitted patients) & Median (days) & $11(7-18)$ & $17(3-28)$ & $\begin{array}{l}\text { Subdistribution HR for live discharge } \\
\text { from ICU }\end{array}$ & $1.293(0.661-2.529)$ & 0.4534 \\
\hline \multicolumn{7}{|l|}{ Mechanical ventilation ( 29 days) } \\
\hline Incidence (all patients) ${ }^{\natural ฯ \uparrow}$ & $\mathrm{CIF}^{\star \prime}(\%)$ & $12.0(6.9-18.6)$ & $15.6(8.0-25.6)$ & Subdistribution $\mathrm{HR}^{\#}$ & $0.738(0.313-1.741)$ & 0.4838 \\
\hline Duration of MV (MV patients) & Median (days) & $13(\mathrm{NC}-\mathrm{NC})$ & 19 (NC-NC) & $\begin{array}{l}\text { Subdistribution HR for live weaning from } \\
\qquad \mathrm{MV}^{\#}\end{array}$ & $0.897(0.263-3.034)$ & 0.8609 \\
\hline \multicolumn{7}{|l|}{ Supplemental oxygen ( 29 days) } \\
\hline Incidence (all patients) & $\mathrm{CIF}^{\text {बI }}(\%)$ & $83.2(75.1-88.9)$ & $79.7(67.4-87.8)$ & Subdistribution $\mathrm{HR}^{\#}$ & $0.998(0.837-1.191)$ & 0.7811 \\
\hline $\begin{array}{l}\text { Duration of supplemental oxygen (patients with } \\
\text { supplemental oxygen)" }\end{array}$ & Median (days) & $6(5-8)$ & $7(5-11)$ & $\begin{array}{l}\text { Subdistribution HR for live weaning from } \\
\text { oxygen" }\end{array}$ & $1.042(0.737-1.479)$ & 0.9465 \\
\hline Hospital stay (29 days) & & & & Subdistribution HR for live & & \\
\hline Occurrence of live hospital discharge & $\mathrm{CIF}^{\bullet}(\%)$ & $85.8(78.0-91.0)$ & $84.4(72.5-91.4)$ & hospital discharge ${ }^{\#}$ & $1.064(0.780-1.451)$ & 0.6954 \\
\hline Duration of hospital stay (days) & Median (days) & $7(6-8)$ & $8(6-9)$ & & & \\
\hline \multicolumn{7}{|l|}{ Safety outcome } \\
\hline $\begin{array}{l}\text { Combined cardiac end-point (hs-troponin } \\
>0.5 \mathrm{ng} \cdot \mathrm{mL}^{-1} \text { and/or ventricular arrhythmia } \\
\text { requiring intervention and/or sudden cardiac } \\
\text { death) }\end{array}$ & $\mathrm{n} / \mathrm{N}(\%)$ & $24 / 119(20.17)$ & $12 / 64(18.75)$ & $\mathrm{OR}^{++}$ & $1.40(0.61-3.19)$ & 0.4265 \\
\hline
\end{tabular}


TABLE 2 Continued

Full analysis set $(n=183)$

Statistic

Estimate $(95 \% \mathrm{Cl})$

Treatment effect

Estimate $(95 \% \mathrm{Cl}) \quad \mathrm{p}$-value AZITHRO SOC

\begin{tabular}{|c|c|c|c|c|c|c|}
\hline \multicolumn{7}{|l|}{ Exploratory outcomes } \\
\hline Normal CT at 5-7 weeks & $\mathrm{n} / \mathrm{N}(\%)$ & 25/75 (33.33) & $12 / 40(30.00)$ & $O R^{\S \S}$ & $1.56(0.64-3.79)$ & 0.3233 \\
\hline$D_{\mathrm{LCO}} \%$ predicted & $\begin{array}{c}\text { Estimated mean } \\
\text { (\%) }\end{array}$ & $66.49(59.96-73.02)$ & $67.00(57.91-76.09)$ & Treatment difference ${ }^{\# \# \#}$ & $0.22(-11.1-11.51)$ & 0.9695 \\
\hline 6-min walk test & $\begin{array}{l}\text { Estimated mean }{ }^{f f} \\
\text { (m) }\end{array}$ & $496.5(465.0-528.0)$ & $456.3(413.2-499.3)$ & Treatment difference ${ }^{\# \# \#}$ & $32.51(-21.2-86.25)$ & 0.2383 \\
\hline
\end{tabular}

AZITHRO: azithromycin; SOC: standard of care; CIF: cumulative incidence functions; HR: hazard ratio; OR: odds ratio; Hosp.: hospitalised; MV: mechanical ventilation; ECMO: extracorporeal membrane oxygenation; KM: Kaplan-Meier; ICU: intensive care unit; NC: not calculated; CT: computed tomography; $D_{\text {Lco: }}$ diffusing capacity of the lung for carbon monoxide. \#: score $>1$ favours azithromycin; HR was obtained using a Fine \& Gray model for competing risk data including treatment, study site and study period as factors. ": event rates were estimated as CIF, taking into account the competing risk of death. ${ }^{+}$: missing data are accounted for by multiple imputation. ${ }^{\S}$ : score $>1$ favours azithromycin; OR was obtained from a multinomial logistic regression with factors for treatment, disease severity and clinical status on day $0 .{ }^{f}$ : geometric mean was obtained using a general linear model including treatment as factor. \#\#: score $>1$ favours SOC; treatment ratio was calculated as the ratio of geometric means, obtained using a general linear model including treatment, study site, study period and clinical status on day 0 as factors (note: data were log-transformed prior to statistical analysis). ${ }^{\uparrow}$ : : score $>1$ favours SOC; HR was obtained using log-rank test after event rates were estimated using Kaplan-Meier methodology. ${ }^{++}$: score $>1$ favours SOC; ORs were obtained using logistic regression including treatment, study site and study period as factors in the model. ${ }^{\S}$ : score $>1$ favours azithromycin; ORs were obtained using

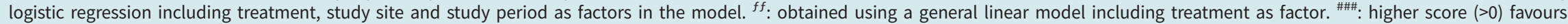
azithromycin; obtained using a general linear model including treatment, study site and period as factors. ${ }^{\text {बฯฯ: }}$ score $>1$ favours SOC; HR was obtained using a Fine \& Gray model for competing risk data including treatment, study site and study period as factors. 


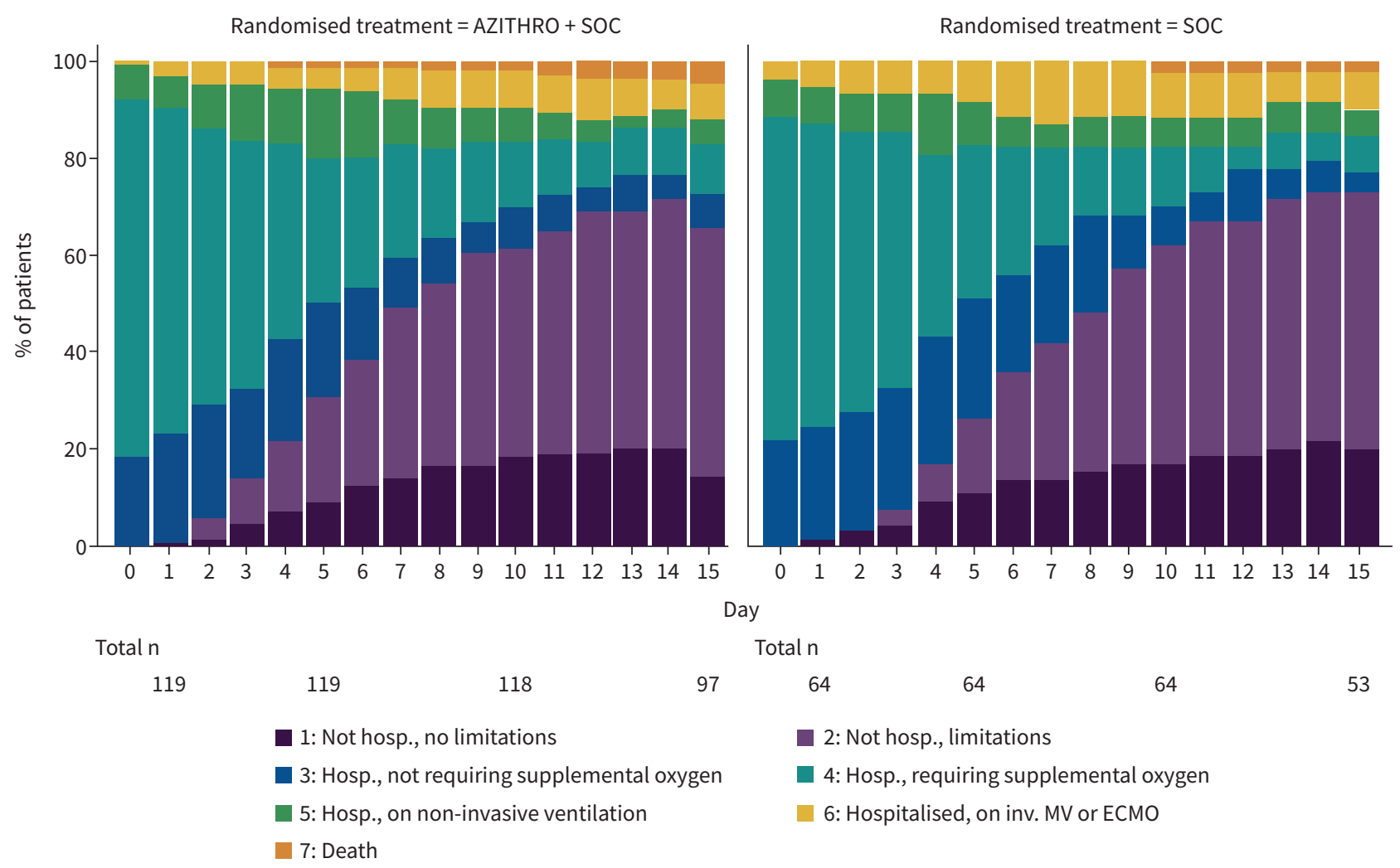

FIGURE 3 Bar chart of daily clinical status (full analysis set). AZITHRO: azithromycin; SOC: standard of care; Hosp.: hospitalised; inv: invasive; MV: mechanical ventilation; ECMO: extracorporeal membrane oxygenation.

bronchiectasis, or chronic lung allograft rejection [23]. In the acute setting of COVID-19, however, azithromycin may miss the potency to benefit. This seems equally true even in mild and early-stage disease, as has recently been shown in studies assessing outpatient treatment with azithromycin [24].

Our trial has some limitations. First, the trial was prematurely stopped after an interim analysis and is therefore underpowered to exclude for a Type II error. However, given the complete overlap of survival curves, the absence of any significant or numerical benefit on secondary outcomes, and the negative evidence on azithromycin for COVID-19 from other trials, the likelihood of wrongly accepting the null hypothesis is extremely low. Second, the trial was open label and not placebo-controlled. This might have led to performance bias in early management, and bias during follow-up and even assessment of the ordinal scale, which is subjective to some degree, both for caregivers (e.g. decision to stop oxygen, discharge) and patients (e.g. perceived degree of limitations at home). Third, the definition of standard of care was not strictly defined, could change according to updated national and international guidelines, and was ultimately left at the treating physician's discretion. This has, however, been corrected by including study period as a covariate in the Fine and Gray regression, showing no significant interaction. Last, no viral outcome was assessed, as other antiviral compounds like remdesivir, which showed much stronger in vitro SARS-CoV-2 antiviral activity than azithromycin [1], had already failed to significantly reduce viral load in a similar population of hospitalised patients [25]. Therefore, eventually, we did not expect the potential antiviral activity of azithromycin to contribute much to the effect size and, at this late disease stage, rather assessed its anti-inflammatory and immunomodulatory effect.

\section{Conclusion}

In summary, we showed that in hospitalised patients with COVID-19, azithromycin did not reduce the time to sustained clinical improvement or discharge. We were also able to confirm a lack of efficacy on clinical status or mortality at the fixed timepoints that were previously assessed by large trials.

Provenance: Submitted article, peer reviewed. 
Acknowledgements: The authors want to thank Maylorie 't Lam, Kaat Haesendonck and Lies Goegebeur for their invaluable contribution to the coordination and data collection of the study.

The DAWn-AZITHRO investigators: T. Van Assche, T. Devos, G. Meyfroidt, H. Ceunen, B. Debaveye, M. 't Lam, K. Haesendonck, L. Goegebeur, J. Neyts, E. Van Wijngaerden and P. De Munter.

This study is registered at https://eudract.ema.europa.eu/ with identifier number 2020-001614-38A. Individual participant data will be available, i.e. individual participant data that underlie the results reported in this article after deidentification. The study protocol and statistical analysis plan will be made available. This information will be available immediately following publication, ending at least 5 years following article publication, for researchers who provide a methodologically sound proposal and to achieve aims in the approved proposal. Proposals should be directed to wim.janssens@kuleuven.be or robin.vos@kuleuven.be. Requestors will need to sign a data access agreement. The data will be available in our University Hospital data warehouse.

Conflict of interest: I. Gyselinck declares funding for the present work from the COVID-19 fund of the KU and UZ Leuven. L. Liesenborghs declares funding for the present work from the COVID-19 fund of the KU and UZ Leuven. A. Belmans declares funding for the present work from the COVID-19 fund of the KU and UZ Leuven. M.M. Engelen declares funding for the present work from the COVID-19 fund of the KU and UZ Leuven. A. Betrains declares funding for the present work from the COVID-19 fund of the KU and UZ Leuven. Q. Van Thillo declares funding for the present work from the COVID-19 fund of the KU and UZ Leuven. P.A.H. Nguyen declares funding for the present work from the COVID-19 fund of the KU and UZ Leuven. P. Goeminne declares payment or honoraria for lectures from AstraZeneca, GlaxoSmithKline and Chiesi, and advisory board participation for Chiesi and GlaxoSmithKline, all in the 26 months prior to manuscript submission; in addition they declare the following unpaid roles: EMBARC board member; BeRS board member; ERS group 10 board member. A-C. Soenen declares funding for the present work from the COVID-19 fund of the KU and UZ Leuven. N. De Maeyer declares funding for the present work from the COVID-19 fund of the KU and UZ Leuven. C. Pilette declares funding for the present work from the COVID-19 fund of the KU and UZ Leuven. E. Papleux declares funding for the present work from the COVID-19 fund of the KU and UZ Leuven. E. Vanderhelst declares funding for the present work from the COVID-19 fund of the KU and UZ Leuven. A. Derweduwen declares funding for the present work from the COVID-19 fund of the $\mathrm{KU}$ and UZ Leuven. P. Alexander declares funding for the present work from the COVID-19 fund of the KU and UZ Leuven. B. Bouckaert declares funding for the present work from the COVID-19 fund of the KU and UZ Leuven. J-B. Martinot declares funding for the present work from the COVID-19 fund of the KU and UZ Leuven. L. Decoster declares funding for the present work from the COVID-19 fund of the KU and UZ Leuven. K. Vandeurzen declares funding for the present work from the COVID-19 fund of the KU and UZ Leuven. R. Schildermans declares funding for the present work from the COVID-19 fund of the KU and UZ Leuven. P. Verhamme declares funding for the present work from the COVID-19 fund of the KU and UZ Leuven. W. Janssens declares participation on an advisory board on antiretrovirals for Gilead Sciences in 2018, and support for attending meetings from Gilead Sciences (EACS 2019) and Sanofi Pasteur (ISTM 2019); in addition, they were secretary of the Belgische Vereniging voor Infectiologie en Klinische Microbiologie until 2020. R. Vos declares funding for the present work from the COVID-19 fund of the KU and UZ Leuven.

Support statement: The study was funded by Life Sciences Research Partners, the COVID-19 fund of the KU Leuven and by the Research Foundation Flanders (G0G4720N). Funding information for this article has been deposited with the Crossref Funder Registry.

\section{References}

1 Liesenborghs L, Spriet I, Jochmans D, et al. Itraconazole for COVID-19: preclinical studies and a proof-of-concept randomized clinical trial. EBioMedicine 2021; 66: 103288.

2 Gyselinck I, Liesenborgs L, Landeloos E, et al. Direct antivirals working against the novel coronavirus: azithromycin (DAWn-AZITHRO), a randomized, multicenter, open-label, adaptive, proof-of-concept clinical trial of new antivirals working against SARS-CoV-2 - azithromycin trial. Trials 2021; 22: 126.

3 Vanassche T, Engelen MM, Van Thillo Q, et al. A randomized, open-label, adaptive, proof-of-concept clinical trial of modulation of host thromboinflammatory response in patients with COVID-19: the DAWn-Antico study. Trials 2020; 21: 1005.

4 Devos T, Van Thillo Q, Compernolle V, et al. Early high antibody titre convalescent plasma for hospitalised COVID-19 patients: DAWn-plasma. Eur Respir J 2022; 59: 2101724.

5 Touret F, Gilles M, Barral K, et al. In vitro screening of a FDA approved chemical library reveals potential inhibitors of SARS-CoV-2 replication. Sci Rep 2020; 10: 13093.

6 Damle B, Vourvahis M, Wang E, et al. Clinical pharmacology perspectives on the antiviral activity of azithromycin and use in COVID-19. Clin Pharmacol Ther 2020; 108: 201-211. 
7 Ishaqui AA, Khan AH, Sulaiman SAS, et al. Assessment of efficacy of Oseltamivir-Azithromycin combination therapy in prevention of Influenza-A (H1N1)pdm09 infection complications and rapidity of symptoms relief. Expert Rev Respir Med 2020; 14: 533-541.

8 Lee N, Wong CK, Chan MCW, et al. Anti-inflammatory effects of adjunctive macrolide treatment in adults hospitalized with influenza: a randomized controlled trial. Antiviral Res 2017; 144: 48-56.

9 Gautret P, Lagier J-C, Parola P, et al. Hydroxychloroquine and azithromycin as a treatment of COVID-19: results of an open-label non-randomized clinical trial. Int J Antimicrob Agents 2020; 56: 105949.

10 Gautret P, Lagier J-C, Parola P, et al. Clinical and microbiological effect of a combination of hydroxychloroquine and azithromycin in 80 COVID-19 patients with at least a six-day follow up: a pilot observational study. Travel Med Infect Dis 2020; 34: 101663.

11 Gyselinck I, Janssens W, Verhamme P, et al. Rationale for azithromycin in COVID-19: an overview of existing evidence. BMJ Open Respir Res 2021; 8: e000806.

12 Cavalcanti $A B$, Zampieri FG, Rosa RG, et al. Hydroxychloroquine with or without azithromycin in mild-to-moderate Covid-19. N Engl J Med 2020; 383: 2041-2052.

13 Furtado RHM, Berwanger O, Fonseca HA, et al. Azithromycin in addition to standard of care versus standard of care alone in the treatment of patients admitted to the hospital with severe COVID-19 in Brazil (COALITION II): a randomised clinical trial. Lancet 2020; 396: 959-967.

14 Abaleke E, Abbas M, Abbasi S, et al. Azithromycin in patients admitted to hospital with COVID-19 (RECOVERY): a randomised, controlled, open-label, platform trial. Lancet 2021; 397: 605-612.

15 Sekhavati E, Jafari F, SeyedAlinaghi SA, et al. Safety and effectiveness of azithromycin in patients with COVID-19: an open-label randomised trial. Int J Antimicrob Agents 2020; 56: 106143.

16 Cao B, Wang Y, Wen D, et al. A trial of Lopinavir-Ritonavir in adults hospitalized with severe Covid-19. N Engl J Med 2020; 382: 1787-1799.

17 Rubin DB. Multiple imputation after 18+ years. J Am Stat Assoc 1996; 91: 473-489.

18 Dodd LE, Follmann D, Wang J, et al. Endpoints for randomized controlled clinical trials for COVID-19 treatments. Clin Trials 2020; 17: 472-482.

19 Venditto VJ, Haydar D, Abdel-Latif A, et al. Immunomodulatory effects of azithromycin revisited: potential applications to COVID-19. Front Immunol 2021; 12: 285.

20 Omrani AS, Pathan SA, Thomas SA, et al. Randomized double-blinded placebo-controlled trial of hydroxychloroquine with or without azithromycin for virologic cure of non-severe Covid-19. EClinicalMedicine 2020; 29: 100645.

21 Lansbury L, Lim B, Baskaran V, et al. Co-infections in people with COVID-19: a systematic review and meta-analysis. J Infect 2020; 81: 266-275.

22 Chertow DS, Memoli MJ. Bacterial coinfection in influenza: a grand rounds review. J Am Med Assoc 2013; 309: 275-282.

23 Parnham MJ, Haber VE, Giamarellos-Bourboulis EJ, et al. Azithromycin: mechanisms of action and their relevance for clinical applications. Pharmacol Ther 2014; 143: 225-245.

24 Butler CC, Dorward J, Yu L-M, et al. Azithromycin for community treatment of suspected COVID-19 in people at increased risk of an adverse clinical course in the UK (PRINCIPLE): a randomised, controlled, open-label, adaptive platform trial. Lancet 2021; 397: 1063-1074.

25 Wang Y, Zhang D, Du G, et al. Remdesivir in adults with severe COVID-19: a randomised, double-blind, placebo-controlled, multicentre trial. Lancet 2020; 395: 1569-1578. 